\section{Letter: Sarcopenia Is Significantly Associated with Presence and Severity of Nonalcoholic Fatty Liver Disease (J Obes Metab Syndr 2019;28:129-38)}

\author{
Hyuktae Kwon* \\ Department of Family Medicine, Seoul National University Hospital, Seoul, Korea
}

\author{
Received November 19, 2019 \\ Reviewed December 14, 2019 \\ Accepted December 16, 2019 \\ ${ }^{*}$ Corresponding author \\ Hyuktae Kwon \\ (D) \\ https://orcid.org/0000-0002-0312-3650 \\ Department of Family Medicine, \\ Seoul National University Hospital, \\ 101 Daehak-ro, Jongno-gu, \\ Seoul 03080, Korea \\ Tel: +82-2-2072-7495 \\ Fax: +82-2-766-3276 \\ E-mail: hyuktae@gmail.com
}

Nonalcoholic fatty liver disease (NAFLD) is one of the most common causes of chronic liver disease in South Korea ${ }^{1}$ and is closely related to abdominal obesity, dyslipidemia, type 2 diabetes mellitus, and metabolic syndrome, all of which are risk factors for cardiovascular disease (CVD). ${ }^{2,3}$ Furthermore, several recently published prospective studies have shown that NAFLD is an independent risk factor for CVD. ${ }^{4-7}$ Insulin resistance is regarded as the major factor contributing to the pathogenesis of NAFLD. ${ }^{8}$ Given that skeletal muscle is one of the important target organs of insulin and results from previous studies showing a link between sarcopenia and insulin resistance, ${ }^{9}$ the study by Chung et al. ${ }^{10}$ is worthy of scientific attention. They have investigated the association between sarcopenia and severity of NAFLD with consideration of various confounders including visceral fat area (VFA) measured by computed tomography scan. Their work has strength in that they performed various stratification analyses and showed that age was a possible modifier of the association between sarcopenia and NAFLD, and that sarcopenia among relatively young ( $<50$ years) adults might have a more detrimental effect on NAFLD.

Although the results were interesting, they did not adjust for possible confounders in the stratification analysis. The main anal- ysis shown in Tables 2 and 3 of Ref. 10 were adjusted for hypertension, diabetes, total cholesterol, and low-density lipoprotein cholesterol, although they considered only sex and VFA in the stratification analysis. As they explained in the Discussion, it would have been better if physical activity had been considered as a possible confounder.

As the authors ${ }^{10}$ suggested insulin resistance as a possible link between sarcopenia and NAFLD, it would be more informative if they could present stratified analysis according to presence of insulin resistance or visceral obesity. Nevertheless, the authors clearly showed the association of sarcopenia and NAFLD, and this association should be confirmed in a longitudinal study.

\section{CONFLICTS OF INTEREST}

The author declares no conflict of interest.

\section{REFERENCES}

1. Park SH, Jeon WK, Kim SH, Kim HJ, Park DI, Cho YK, et al. Prevalence and risk factors of non-alcoholic fatty liver disease among Korean adults. J Gastroenterol Hepatol 2006;21(1 Pt 
1):138-43.

2. Moscatiello S, Manini R, Marchesini G. Diabetes and liver disease: an ominous association. Nutr Metab Cardiovasc Dis 2007;17:63-70.

3. Bedogni G, Gastaldelli A, Manco M, De Col A, Agosti F, Tiribelli $\mathrm{C}$, et al. Relationship between fatty liver and glucose metabolism: a cross-sectional study in 571 obese children. Nutr Metab Cardiovasc Dis 2012;22:120-6.

4. Lin YC, Lo HM, Chen JD. Sonographic fatty liver, overweight and ischemic heart disease. World J Gastroenterol 2005;11: 4838-42.

5. Hamaguchi M, Kojima T, Takeda N, Nagata C, Takeda J, Sarui $\mathrm{H}$, et al. Nonalcoholic fatty liver disease is a novel predictor of cardiovascular disease. World J Gastroenterol 2007;13:1579-84.

6. Volzke H, Robinson DM, Kleine V, Deutscher R, Hoffmann W, Ludemann J, et al. Hepatic steatosis is associated with an in- creased risk of carotid atherosclerosis. World J Gastroenterol 2005;11:1848-53.

7. Choi SY, Kim D, Kim HJ, Kang JH, Chung SJ, Park MJ, et al. The relation between non-alcoholic fatty liver disease and the risk of coronary heart disease in Koreans. Am J Gastroenterol 2009; 104:1953-60.

8. Machado M, Cortez-Pinto H. Non-alcoholic steatohepatitis and metabolic syndrome. Curr Opin Clin Nutr Metab Care 2006;9:637-42.

9. Guillet C, Boirie Y. Insulin resistance: a contributing factor to age-related muscle mass loss? Diabetes Metab 2005;31:5S20$5 S 26$.

10. Chung GE, Kim MJ, Yim JY, Kim JS, Yoon JW. Sarcopenia is significantly associated with presence and severity of nonalcoholic fatty liver disease. J Obes Metab Syndr 2019;28:129-38. 